Terr. Atmos. Ocean. Sci., Vol. 18, No. 4, 827-841, October 2007

\title{
Error Propagation From Aerosol Retrieval to Atmospheric Correction Due to Adjacency Effect for FORMOSAT-2 RSI Band
}

\author{
Chien-Hui Liu ${ }^{1, \text { * }}$
}

(Manuscript received 16 January 2006, in final form 2 March 2007)

\begin{abstract}
Homogeneous surface is usually assumed in aerosol retrieval for the dark target method. Retrieved aerosol optical depth can thus be overestimated. This error will also be propagated into retrieved surface reflectance during atmospheric correction, especially for high spatial resolution of $8 \mathbf{m}$ for FORMOSAT-2 (also known as ROCSAT-2) RSI. The error in retrieved surface reflectance during atmospheric corrections induced by retrieved aerosol optical depth error due to adjacency effect over a dark target is studied in the green and red bands of FORMOSAT-2 RSI. The results show that significant errors in retrieved surface reflectance, i.e., larger than 0.01, may occur in most cases except at low contrast for a bright target. Such an error for a dark target is larger than that for a bright target for given contrast and haziness. Relatively, the error of retrieved surface reflectance is enhanced by the introduced error of aerosol optical depth in atmospheric correction for dark targets. The error can be larger than $35 \%$ for dark targets and less than $\mathbf{1 0 \%}$ for bright targets. Hence, it is suggested that adjacency effect be considered from aerosol optical depth retrieval to surface reflectance retrieval in atmospheric correction except at low contrast for bright targets.
\end{abstract}

(Key words: Adjacency effect, Aerosol optical depth, Atmospheric correction, FORMOSAT-2 RSI)

\footnotetext{
${ }^{1}$ Department of Information Management/Geographic Information Management and Research Center, Transworld Institute of Technology, Yunlin, Taiwan, ROC

* Corresponding author address: Prof. Chien-Hui Liu, Department of Information Management/Geographic Information Management and Research Center, Transworld Institute of Technology, Yunlin, Taiwan, ROC; E-mail: chliu@tit.edu.tw doi: 10.3319/TAO.2007.18.4.827(AA)
} 


\section{INTRODUCTION}

FORMOSAT-2 (also known as ROCSAT-2) developed by the National SPace Organization (NSPO) of Taiwan was successfully launched on 21 May 2004 (http://www.nspo.gov.tw). Remote sensing instrument (RSI) on board can provide images of high spatial resolution of $2 \mathrm{~m}$ in panchromatic band and $8 \mathrm{~m}$ in four multispectral bands (Lee et al. 2002) with central wavelengths of $0.484,0.561,0.661$, and $0.821 \mu \mathrm{m}$, respectively (Liu 2005). Its mission is to image Taiwan and the surrounding areas on a daily basis for various applications, such as environmental monitoring, natural resources research, disaster prevention, and rescue work. Since scattering and absorption in the atmosphere can distort the received signal from the satellite, atmospheric correction (AC) is necessary to convert the top-of-atmosphere (TOA) radiance to surface reflectance for analysis of remotely sensed data quantitatively (Kaufman 1989; Liang et al. 2001; Liang et al. 2002) such as leaf area index retrieval (Butson and Fernandes 2004), forest mapping (Pax-Lenney et al. 2001), crop detection (Sakamoto et al. 2005), and water quality monitoring (Pozdnyakov et al. 2005).

To correct the atmospheric effect, the optical characteristics of the atmosphere should be first estimated. Molecular scattering and ozone absorption can be considered to have an invariant effect and be accounted for satisfactorily. The absorption of water vapor can be significant for RSI's near-infrared band. Its accuracy can be improved if radiosonde data are available (Liu et al. 1996); however, climatology data or other satellite output can be applied in practice (Liang et al. 2001). Owing to the nature of temporal and spatial variations, aerosol effect is the most difficult part of AC to correct. Aerosol characteristics, such as aerosol optical depth (AOD), size distribution and single scattering albedo, can be observed and determined from sunphotometer (Holben et al. 2001; Dubovik et al. 2002). Suitable aerosol models can also be chosen by geo-location (Kaufman et al. 1997). The image-based method seems to be the only way to retrieve AOD for operational AC. It has also been developed in numerous previous studies (Kaufman and Sendra 1988; Liang et al. 1997; Ouaidrari and Vermote 1999; Liang et al. 2001; Vermote et al. 2002; Lyapustin et al. 2004). After these parameters are known, retrieval of surface reflectance can be very straightforward (Kaufman 1989).

Currently, the most promising operational algorithm for global and regional AOD retrieval over land is the dark target (DT) method (Kaufman and Sendra 1988). This method has been modified to take advantage of the low opacity of most aerosol types in the mid-IR bands, and the high correlation of surface reflectance between visible and mid-IR bands for the EOS moderate resolution imaging spectroradiometer (MODIS) (Kaufman et al. 1997) and Landsat TM/ETM+ (Liang et al. 1997; Ouaidrari and Vermote 1999). The DT can be identified as pixels with low near-IR signal and high vegetation index (Kaufman and Sendra 1988) for sensors without mid-IR bands, such as SPOT HRV and FORMOSAT-2 RSI. In this case, reasonable reflectance of DT can then be assumed in the visible bands. This is because the TOA signal contains larger atmospheric reflectance and surface reflectances are very low in the visible bands for DTs, the errors of retrieved AOD due to the errors of the assumed reflectances over DTs are expected to be much lower than those over bright targets. DTs are mainly dense vegetation and dark soils (Kaufman et al. 1997). The main sources of errors for the DT method are surface inhomogeneity and sub-pixel water contamination (Chu et al. 2002; Levy et al. 2005). 
Classical 1-D radiative transfer (RT) theory, which assumes the surface to be infinite and uniform, is usually used in aerosol retrieval for the DT method. In fact, the interaction of atmospheric scattering and heterogeneous surface can systematically brighten the DT and darken the bright target (BT) at the TOA level (Lyapustin 2001; Lyapustin and Kaufman 2001; Lyapustin et al. 2004). This is caused by the so-called adjacency effect. Thus retrieved AOD from DT will be overestimated. The error is significant even at $1 \mathrm{~km}$ resolution (Lyapustin and Kaufman 2001). It is reported that the error of retrieved AOD at 30-m resolution for the red wavelength can exceed $100 \%$ over a medium surface at nadir view in clear sky. For FORMOSAT-2 RSI with 8-m spatial resolution, the errors of retrieved AOD can be about $100 \%$ and $97 \%$ for the blue and red bands, respectively, over a medium surface at a viewing zenith angle of $45^{\circ}$ in clear sky (Liu 2005). As can be shown later in this study, error increases when the viewing angle moves to nadir. It is also shown that this error is larger for urban models than that for continental models. This AOD error can further introduce error into surface retrieval in AC. These errors are increased as spatial resolution increases. Therefore, it is suggested that adjacency effect (AE) be taken into account not only in the AC algorithms but also in aerosol retrieval over land even at a sensor resolution of $1 \mathrm{~km}$ (Lyapustin and Kaufman 2001). In spite of 1-D RT theory assumed by operational algorithms (Kaufman et al. 1997; Liang et al. 1997; Ouaidrari and Vermote 1999), a new DT method of aerosol retrieval based on 3-D RT theory for the Landsat enhanced thematic mapper plus (ETM+) has been developed (Lyapustin et al. 2004) recently. It simultaneously retrieves the aerosol model and AOD with excellent accuracy $( \pm 0.02-0.03)$ in the Washington-Baltimore area. Surface climatology considering seasonal and geographic variations of regression coefficients between visible and mid-IR reflectances is proposed in this method. It may be helpful for aerosol retrieval from RSI data, since the spectral characteristics of RSI are very similar to those of ETM+ (Liu 2005), even though there is a lack of mid-IR band.

Based on the previous study (Liu 2005), error analysis of retrieved surface reflectance caused by the error of retrieved AOD due to AE for FORMOSAT-2 RSI with its high spatial resolution of $8 \mathrm{~m}$ is presented in this paper. The reasons why these errors occur are also explained with their physical mechanisms. For comparison purposes with the results of Lyapustin and Kaufman (2001), some parameters are set to the same. Because of the lower sensitivity of TOA reflectance to AOD for the more absorptive urban aerosol model and the usual absence of feasible AOD to fit $\rho_{T O A}$ for medium contrast (Liu 2005), only the continental aerosol model is discussed in this study. The analysis is performed in the green and red bands. The error with viewing zenith angle is also discussed.

\section{METHODOLOGY}

A simple AC model (SACM) (Liu 2003), previously used to estimate the error in AOD due to AE over a DT (Liu 2005), is applied to determine the error of retrieved surface reflectance $\rho_{c}$ in AC for ROCSAT-2 RSI. The SACM is based on the second simulation of the satellite signal in the solar spectrum (6S)(Vermote et al. 1997), which is accurate and has been successfully used in the development of an atmospheric correction model for EOS-MODIS 
data (Vermote et al. 2002). The gaseous transmission and Rayleigh optical depth are simplified as analytic functions. Lookup tables are compiled to determine Rayleigh and aerosol scattering. The environment function is defined as the probability of scattered photons reaching the sensor from the assumed uniform circular target over an inhomogeneous surface in 6S. Its lookup table is compiled with a radius of $4 \mathrm{~m}$ in SACM, i.e., half of spatial resolution of ROCSAT-2 RSI. The rmse values of TOA reflectances $\rho_{T O A}$ simulated by SACM are 0.0008 and 0.0006 in blue and red bands, respectively, for the continental aerosol model, when compared with $6 \mathrm{~S}$ for a wide range of parameters. The major contribution of SACM is that it not only quite accurately reproduces TOA reflectance, but also does so faster than 6S (Liu 2003). A similar approach is also applied for SPOT HRV data (Liu 2004). Numerical studies have been conducted to estimate the induced errors of retrieved AOD $\tau_{a}$ due to the rmse of $\rho_{T O A}$ by SACM. A DT with the reflectance $\rho_{c}$ of 0.01 in the red band is assumed at nadir view and solar zenith angle of $30^{\circ}$. Two contrasts over DT are considered, according to Lyapustin and Kaufman (2001). The low contrast (LC) and medium contrast (MC) denote that DT is surrounded by the background with its reflectance $\rho_{b}=0.06$ and $\rho_{b}=0.11$, respectively. The results show that the induced errors are only 0.013 and 0.027 in clear sky $\left(\tau_{a}=0.23\right)$ and hazy sky $\left(\tau_{a}=0.76\right)$ for MC, respectively, for the continental aerosol model. Such an error in AOD can only further introduce the errors in surface reflectance by 0.0009 and 0.0018 , respectively. Errors in AOD and introduced error in surface reflectance are much less than 0.1 and 0.01 . Hence, these errors are neglected in the following discussion.

To better understand both processes of AOD retrieval and its application to AC of $\rho_{T O A}$, let us simply consider $\rho_{T O A}=\rho_{T O A}\left(\tau_{a}, \rho_{c}, \rho_{b}\right)$ by neglecting its dependence of altitude z, solar zenith angle $\theta_{s}$, viewing zenith angle $\theta_{v}$ and viewing azimuth angle $\phi$ for given aerosol model. The whole process includes three steps: (1) $\rho_{T O A}$ is simulated with the true AOD $\tau_{a}$ and $\rho_{c}$ as well as $\rho_{b}$ by considering AE; (2) the estimated AOD $\tau_{a}^{\prime}$ over the DT is retrieved by inverting $\rho_{T O A}$ with the assumption of uniform surface $\left(\rho_{b}=\rho_{c}\right)$, which means $\tau_{a}^{\prime}$ is retrieved by neglecting AE; (3) the estimated surface reflectance $\rho_{c}$ is determined by inverting $\rho_{T O A}$ with $\tau_{a}$ and $\rho_{b}$, which represents the error in $\tau_{a}$ can be potentially propagated into $\rho_{c}$. Hence, the errors in estimated surface reflectance in $\mathrm{AC}$ due to the errors in retrieved AOD by neglecting $\mathrm{AE}$ can be estimated. Meanwhile, the mean absolute and relative errors in the estimated surface reflectance in $\mathrm{AC}$ are determined by considering these errors for different contrasts, haziness and $\theta_{v}$. In numerical computation, it is worth noting that in step (2), AOD is interpolated from the lookup table of $\rho_{T O A}\left(\tau_{a}\right)$ (Kaufman and Sendra 1988); in step (3), surface reflectance is determined by computation of the analytic function of gas transmittance as well as interpolation of lookup tables of Rayleigh and aerosol scattering for given $\theta_{s}, \theta_{v}$, and $\phi$ (Ouaidrari and Vermote 1999; Liu 2004).

Numerical simulations under different conditions are conducted using the aforementioned processes. Both $\tau_{a}$ values of 0.23 and 0.76 at $550 \mathrm{~nm}$ are considered. The values of $\theta_{s}$ are set to be $30^{\circ}$ and $60^{\circ}$, and $\theta_{v}$ varies from $0^{\circ}$ to $45^{\circ}$. The values of $\phi$ are set to be $0^{\circ}$ (backscattering) and $180^{\circ}$ (forward-scattering). Only DTs, i.e., $0.01 \leq \rho_{c} \leq 0.06$ (Lyapustin and Kaufman 2001), are considered in the retrieval of AOD; however, targets with $\rho_{c}$ ranging from 0.01 to 0.21 are considered in AC. The values of $\rho_{b}$ vary from 0.01 to 0.21 in aerosol retrieval and AC. As 
mentioned above, two contrasts over the DT including LC with $\rho_{b}$ of 0.06 and $\mathrm{MC}$ with $\rho_{b}$ of 0.11 are considered, according to Lyapustin and Kaufman (2001).

\section{RESULTS AND DISCUSSION}

Before estimation of the errors in $\rho_{c}$ for AC, AOD retrieval over the DT is performed under the assumption of homogeneous surface. The errors in the retrieved AOD are reported in green and red bands for different haziness, $\theta_{v}$ and contrasts (Table 1a, b). The values of $\theta_{s}$ and $\phi$ are $30^{\circ}$ and $0^{\circ}$, respectively. The mean absolute and relative errors $\varepsilon_{A}$ and $\varepsilon_{R}$ are determined from the errors over the DT with $\rho_{b}$ ranging from 0.01 to 0.21 . As indicated in the previous study (Liu 2005), $\varepsilon_{A}\left(\tau_{a}\right)$ increases as the AOD increases and/or the contrast increases. This is because of neglecting stronger scattered radiance due to the homogeneous surface assumption. Tables $1 \mathrm{a}$ and $\mathrm{b}$ also show that $\varepsilon_{A}\left(\tau_{a}\right)$ decreases as $\theta_{v}$ increases. This is due to the fact that atmospheric reflectance increases and the proportion of the scattering attributed to adjacent surface decreases when $\theta_{v}$ increases. However, compared with the sensitivity to contrast and $\mathrm{AOD}, \varepsilon_{A}\left(\tau_{a}\right)$ is less sensitive to $\theta_{v}$. In fact, one can see that $\varepsilon_{A}\left(\tau_{a}\right)$ is most sensitive to contrast, which is consistent with the results of Lyapustin and Kaufman (2001), and least sensitive to $\theta_{v}$ for the ranges of three parameters discussed. In both bands, $\varepsilon_{A}\left(\tau_{a}\right)$ can exceed 0.1 for LC in hazy sky and 0.2 for MC in both skies. Even for LC in clear sky, $\varepsilon_{A}\left(\tau_{a}\right)$ can be 0.07 at nadir. Although the values of $\varepsilon_{A}\left(\tau_{a}\right)$ in both bands differ only less than 0.05 , the induced $\varepsilon_{A}\left(\rho_{c}\right)$ in the green band can be up to 0.05 greater than that in the red band, which will be discussed later. Hence $\varepsilon_{A}\left(\tau_{a}\right)$ can be quite significant especially for MC and/or hazy sky. As for $\varepsilon_{R}\left(\tau_{a}\right)$, it increases as AOD decreases and/or contrast increases. $\varepsilon_{R}\left(\tau_{a}\right)$ also decreases with $\theta_{v} \cdot \varepsilon_{R}\left(\tau_{a}\right)$ can be at least $16 \%$ for LC in hazy sky at $\theta_{v}=45^{\circ}$ and up to $105 \%$ for $\mathrm{MC}$ in clear sky at nadir in the red band. The latter error seems to be reasonable, since this error at 30-m spatial resolution is reported to exceed $100 \%$ for the same conditions (Lyapustin and Kaufman 2001). In the green band, the values of $\varepsilon_{R}\left(\tau_{a}\right)$ ranging from $15 \%$ to $103 \%$ are similar to that in the red band. These results show the need to consider AE in aerosol retrieval especially for MC.

The error in retrieved AOD due to AE may cause an error in derived surface reflectance in $\mathrm{AC}$, since surface reflectance is retrieved by inverting $\rho_{T O A}$ with the same parameters given in the simulation of $\rho_{T O A}$, except for the retrieved AOD. As pointed out by Fraser and Kaufman (1985) and Kaufman (1989), critical reflectance (CR) is defined as the surface reflectance where $\rho^{T O A}$ stays essentially constant as $\tau_{a}$ increases. CR depends on aerosol optical characteristics, sun and viewing geometry. At CR, as $\tau_{a}$ increases, the amount of increase in atmospheric reflectance due to more aerosol backscattering is equal to that of the decrease in surface reflection owing to decreased atmospheric transmittance. The amount of decrease in the surface reflection is weighted by surface reflectance. For $\rho_{c}<\mathrm{CR}, \rho^{T O A}$ increases with increasing $\tau_{a}$, and for $\rho_{c}>\mathrm{CR}, \rho^{T O A}$ decreases with increasing $\tau_{a}$. As mentioned above, CR has been defined as the surface reflectance where $\rho^{T O A}$ stays essentially constant with varying $\tau_{a}$. To see the performance of CR under a heterogeneous surface assumption, $\rho^{T O A}$ is plotted as a function of $\rho_{c}$ and $\rho_{b}$ for different $\tau_{a}$ in the red band of FORMOSAT-2 RSI (Fig. 1). The 
Table 1. The mean absolute and relative errors ( $\%$ in parenthesis), i.e., $\varepsilon_{A}$ and $\varepsilon_{R}$, of the retrieved $\tau_{a}$ due to $\mathrm{AE}$ and $\rho_{c}$ in AC for different viewing zenith angle $\theta_{v}$, haziness and contrasts in the (a) green and (b) red bands of FORMOSAT-2 RSI. The retrieval of AOD is only for a DT ( $\rho_{c}=0.01 \sim$ 0.06). The low and medium contrast with a DT surrounded by $\rho_{b}=0.06$ and $\rho_{b}=0.11$ are denoted by LC and MC, respectively. Clear and hazy skies correspond to the $\tau_{a}$ values of 0.23 and 0.76 , respectively. $\theta_{s}$ and $\phi$ are $30^{\circ}$ and $0^{\circ}$, respectively. The errors of retrieved $\rho_{c}$ are discussed with respect to DTs and bright targets (BTs), i.e., $\rho_{c}=0.16 \sim 0.21$.

(a) Green band

\begin{tabular}{|c|c|c|c|c|c|}
\hline \multirow{2}{*}{\multicolumn{2}{|c|}{ Mean error }} & \multicolumn{2}{|c|}{$\mathrm{LC}$} & \multicolumn{2}{|c|}{$\mathrm{MC}$} \\
\hline & & clear & hazy & clear & hazy \\
\hline \multicolumn{6}{|c|}{$\theta_{v}=0^{\circ}$} \\
\hline \multicolumn{2}{|c|}{$\varepsilon_{A}\left(\tau_{a}\right)\left[\varepsilon_{R}\left(\tau_{a}\right)\right]$} & $0.07(32)$ & $0.13(16)$ & $0.23(103)$ & $0.41(53)$ \\
\hline \multirow{2}{*}{$\varepsilon_{A}\left(\rho_{c}\right)\left[\varepsilon_{R}\left(\rho_{c}\right)\right]$} & DT & $0.010(44)$ & $0.027(118)$ & $0.036(161)$ & $0.111(481)$ \\
\hline & $\mathrm{BT}$ & $0.005(3)$ & $0.003(2)$ & $0.015(8)$ & $0.012(6)$ \\
\hline \multicolumn{6}{|c|}{$\theta_{v}=45^{\circ}$} \\
\hline \multicolumn{2}{|c|}{$\varepsilon_{A}\left(\tau_{a}\right)\left[\varepsilon_{R}\left(\tau_{a}\right)\right]$} & $0.06(27)$ & $0.11(15)$ & $0.19(85)$ & $0.36(47)$ \\
\hline \multirow{2}{*}{$\varepsilon_{A}\left(\rho_{c}\right)\left[\varepsilon_{R}\left(\rho_{c}\right)\right]$} & DT & $0.013(58)$ & $0.041(178)$ & $0.048(211)$ & $0.176(757)$ \\
\hline & BT & $0.004(2)$ & $0.008(5)$ & $0.012(6)$ & $0.048(27)$ \\
\hline
\end{tabular}

(b) Red band

\begin{tabular}{|c|c|c|c|c|c|}
\hline \multirow{2}{*}{\multicolumn{2}{|c|}{ Mean error }} & \multicolumn{2}{|c|}{$\mathrm{LC}$} & \multicolumn{2}{|c|}{$\mathrm{MC}$} \\
\hline & & Clear & hazy & clear & hazy \\
\hline \multicolumn{6}{|c|}{$\theta_{v}=0^{\circ}$} \\
\hline \multicolumn{2}{|c|}{$\varepsilon_{A}\left(\tau_{a}\right)\left[\varepsilon_{R}\left(\tau_{a}\right)\right]$} & $0.07(33)$ & $0.14(18)$ & $0.24(105)$ & $0.45(59)$ \\
\hline \multirow{2}{*}{$\varepsilon_{A}\left(\rho_{c}\right)\left[\varepsilon_{R}\left(\rho_{c}\right)\right]$} & DT & $0.008(35)$ & $0.021(93)$ & $0.027(124)$ & $0.084(370)$ \\
\hline & BT & $0.004(2)$ & $0.004(2)$ & $0.015(8)$ & $0.012(6)$ \\
\hline \multicolumn{6}{|c|}{$\theta_{v}=45^{\circ}$} \\
\hline \multicolumn{2}{|c|}{$\varepsilon_{A}\left(\tau_{a}\right)\left[\varepsilon_{R}\left(\tau_{a}\right)\right]$} & $0.06(27)$ & $0.12(16)$ & $0.19(85)$ & $0.38(50)$ \\
\hline \multirow{2}{*}{$\varepsilon_{A}\left(\rho_{c}\right)\left[\varepsilon_{R}\left(\rho_{c}\right)\right]$} & DT & $0.01(43)$ & $0.031(133)$ & $0.035(155)$ & $0.125(542)$ \\
\hline & BT & $0.004(2)$ & $0.004(2)$ & $0.012(6)$ & $0.018(10)$ \\
\hline
\end{tabular}




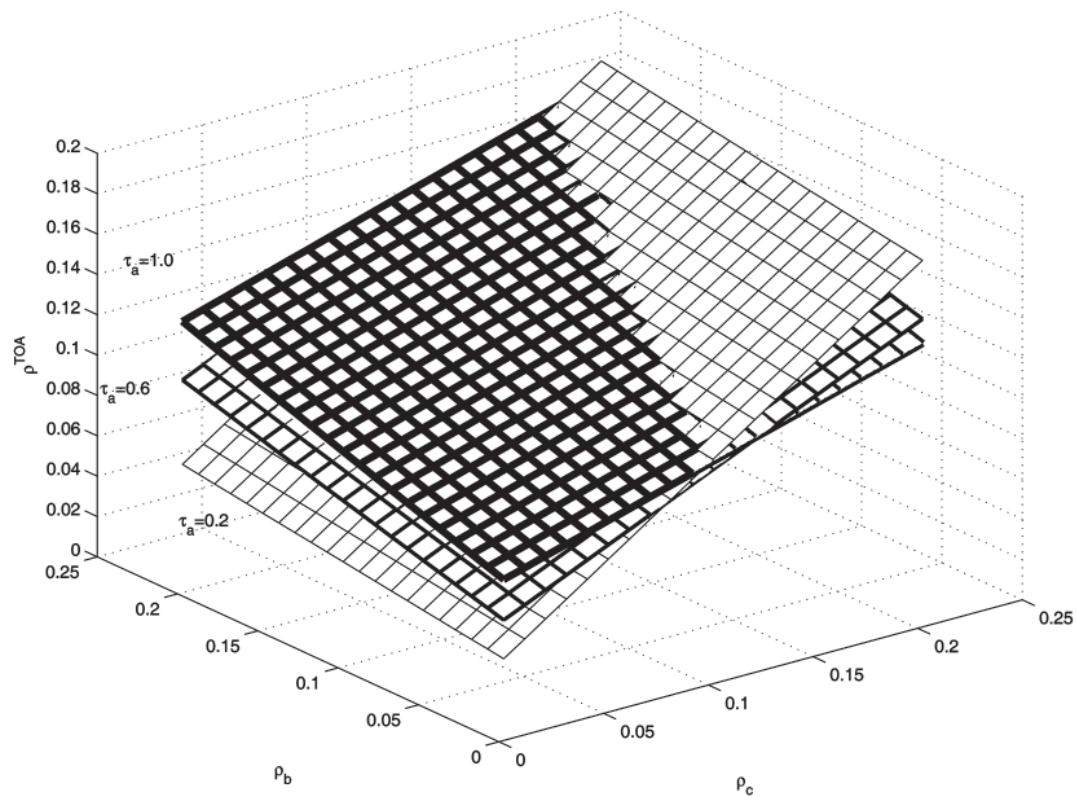

Fig. 1. $\rho^{T O A}$ as a function of $\rho_{c}$ and $\rho_{b}$ for different aerosol optical depth $\tau_{a}$ in the red band of FORMOSAT-2 RSI. The values of solar zenith angle $\theta_{s}$ and viewing zenith angle $\theta_{v}$ are $30^{\circ}$ and $0^{\circ}$, respectively.

values of $\theta_{s}$ and $\theta_{v}$ are $30^{\circ}$ and $0^{\circ}$, respectively. CR increases as $\rho_{b}$ increases. In fact CR is not along the intersected line of $\rho^{T O A}$ for various $\tau_{a}$. Figure 2a, b, and c illustrate the cases as in Fig. 1 but for special $\rho_{b}$ in the red band. For $\rho_{b}=0.01$ (Fig. 2a), CR locates in the region among 0.09 and 0.14 , and for $\rho_{b}=0.21$ (Fig. 2c), CR locates around 0.19. For homogeneous surface $\left(\rho_{b}=\rho_{c}\right)$ (Fig. 2b), CR locates around 0.17. Since the cases for special $\rho_{b}$ in the green band are similar to those in the red band, their figures are omitted; however, quantitative results are reported. CR locates in the region among 0.10 and 0.16 for $\rho_{b}=0.01$, around 0.19 for $\rho_{b}=0.21$, and 0.17 for $\rho_{b}=\rho_{c}$ in the green band. Therefore, CR increases as $\tau_{a}$ increases for low $\rho_{b}$ in both bands. One can then see that $\mathrm{CR}$ locates somewhat in a triangular region whose base is at low $\rho_{b}$ and vertex is at high $\rho_{b}$. This region is called the critical reflectance region (CRR) here. Hence CR will be represented as the critical reflectance inside the CRR in the following discussion. Based on this study, when positive bias occurs on $\tau_{a}$ retrieval due to AE over a DT, $\rho_{c}$ will be underestimated for $\rho_{c}<\mathrm{CR}$, and it will be overestimated for $\rho_{c}>\mathrm{CR}$ as indicated in Fig. 3. The contour lines show the errors in retrieved surface reflectance $\delta \rho_{c}$ caused by the errors in retrieved AOD $\delta \tau_{a}$ due to AE for different $\rho_{c}$ and $\rho_{b}$ in the red band of FORMOSAT-2 RSI. The values of $\theta_{s}$ and $\theta_{v}$ are $30^{\circ}$ and $0^{\circ}$, respectively. The AOD has been retrieved over a dark target ( $\rho_{c}=0.03$ ) under the assumption of homogeneous surface for different contrasts and haziness. As discussed above about the sensitivity of retrieved AOD 
error to contrast and haziness, $\delta \tau_{a}$ is largest, i.e., 0.47, for hazy sky and MC (Fig. 3d); and it is smallest, i.e., 0.09 , for clear sky and LC (Fig. 3a). For given $\rho_{c}, \delta \rho_{c}$ decreases as $\rho_{b}$ increases for both contrasts and haziness. It is because increased adjacent scattering needs to be compensated by decreased surface reflection. For example, as $\rho_{b}$ increases from 0.01 to 0.21 for $\rho_{c}$ of $0.03, \delta \rho_{c}$ decreases from -0.005 to -0.015 for clear sky and LC and -0.083 to -0.102 for hazy sky and MC. For given $\rho_{b}, \delta \rho_{c}$ increases as $\rho_{c}$ increases for both contrasts and haziness. This is because when $\rho_{c}$ increases, the attenuation of surface reflection due to the positive bias $\delta \tau_{a}$ increases. Thus it needs to be compensated by increased $\rho_{c}$. For example, as $\rho_{c}$ increases from 0.01 to 0.21 for $\rho_{b}$ of $0.15, \delta \rho_{c}$ increases from -0.014 to 0.006 for clear sky and LC and -0.109 to 0.020 for hazy sky and MC. The values of $\delta \rho_{c}$ range from -0.017 to 0.013 for clear sky and LC and -0.115 to 0.035 for hazy sky and MC in the red band. In the green band (Fig. 4), the behavior of $\delta \rho_{c}$ is similar to that in the red band. The difference of $\delta \rho_{c}$ in both bands is insignificant, i.e., less than 0.01, in most regions of $\rho_{c}$ and $\rho_{b}$ for LC in both skies and MC in clear sky. However, the magnitude of $\delta \rho_{c}$ is more than 0.01 larger than that in the red band in most regions of $\rho_{c}$ and $\rho_{b}$ for hazy sky and MC This is because a larger increase in atmospheric reflectance in the green band, in spite of comparable $\delta \tau_{a}$ in both bands for this case. As a result, significant $\delta \rho_{c}$ in AC can be caused by $\delta \tau_{a}$ due to AE, especially over a DT for hazy sky and/or MC.
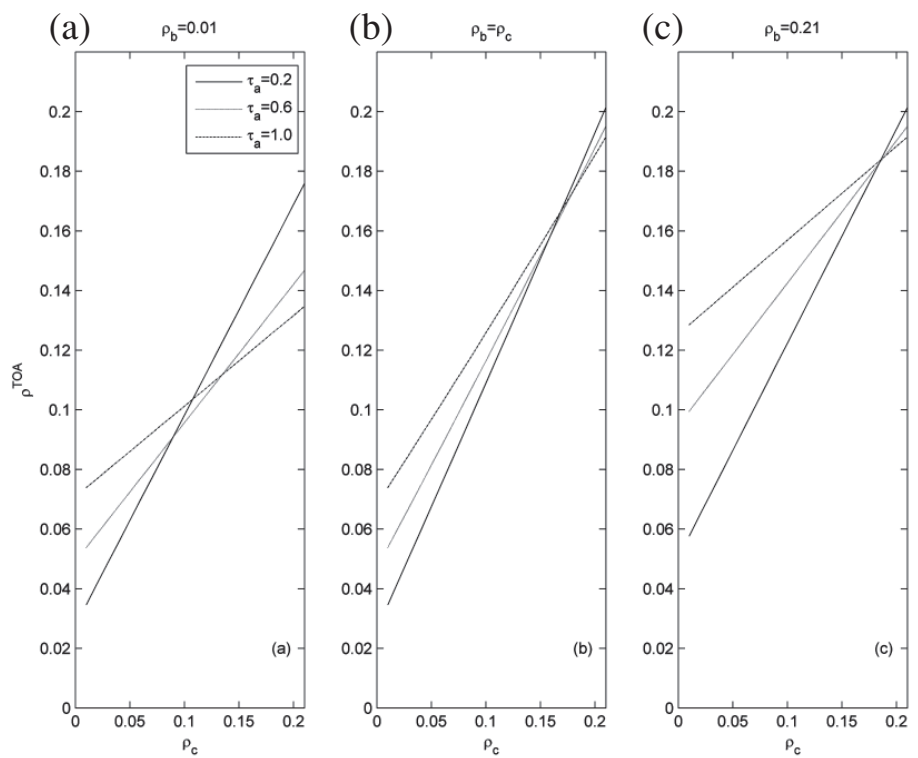

Fig. 2. Top-of-atmospheric reflectance $\rho^{T O A}$ as a function of surface reflectance $\rho_{c}$ for different aerosol optical depths $\tau_{a}$ and background reflectances $\rho_{b}$ in the red band of FORMOSAT-2 RSI. (a), (b), and (c) are $\rho_{b}=0.01$, $\rho_{b}=\rho_{c}$, and $\rho_{b}=0.21$, respectively. The values of $\theta_{s}$ and $\theta_{v}$ are $30^{\circ}$ and $0^{\circ}$, respectively. 

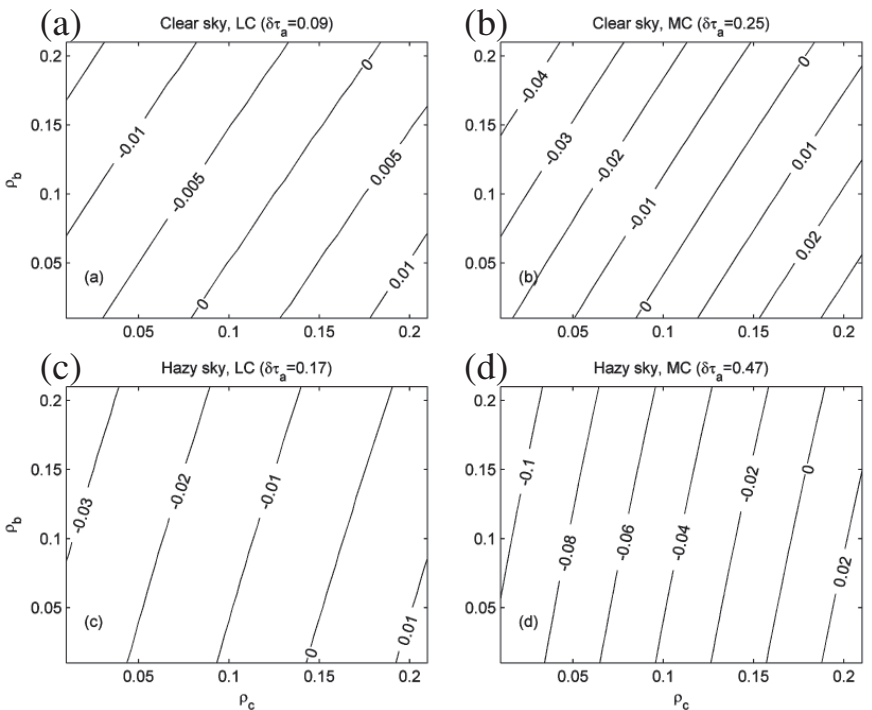

Fig. 3. Error in retrieved surface reflectance caused by the error in retrieved AOD $\delta \tau_{a}$ due to AE for different surface reflectance $\rho_{c}$ and background reflectance $\rho_{b}$ in the red band of FORMOSAT-2 RSI. The values of AOD are retrieved over a DT ( $\left.\rho_{c}=0.03\right)$ by neglecting AE for different haziness and contrasts, including: (a) clear sky and LC, (b) clear sky and MC, (c) hazy sky and LC, and (d) hazy sky and MC. The values of solar zenith angle $\theta_{s}$ and viewing zenith angle $\theta_{v}$ are $30^{\circ}$, and $0^{\circ}$, respectively.
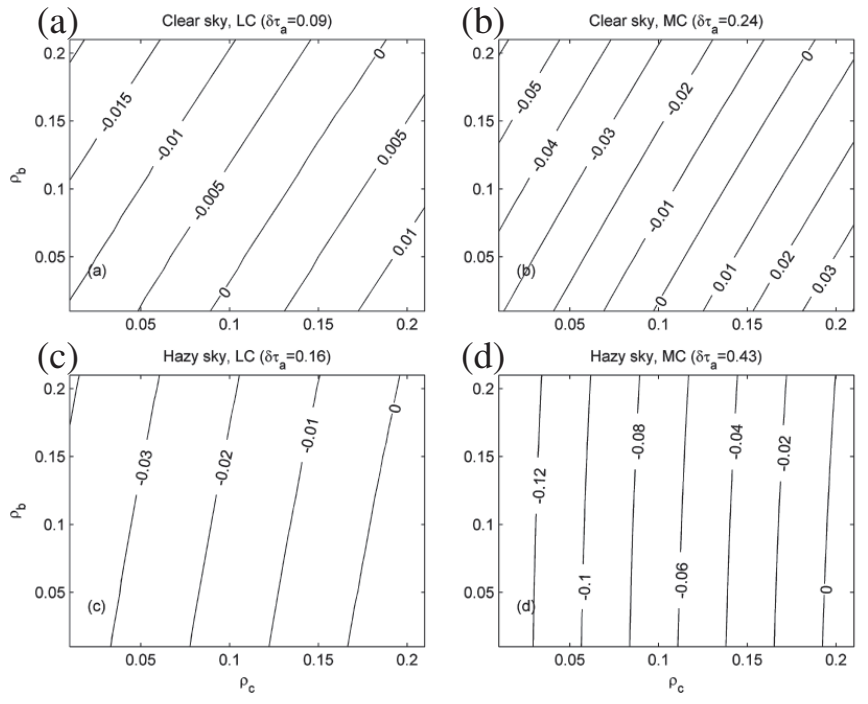

Fig. 4. Similar to Fig. 3, except in green band of FORMOSAT-2 RSI. 
To better understand these errors of retrieved surface reflectance in general, mean absolute errors in retrieved surface reflectance $\varepsilon_{A}\left(\rho_{c}\right)$ due to $\delta \tau_{a}$ for different contrasts and haziness as a function of $\rho_{c}$ in the red band of FORMOSAT-2 RSI are illustrated in Fig. 5. The viewing direction is in nadir and $\theta_{s}$ is $30^{\circ}$. For given contrast and haziness, when $\rho_{c}$ increases, $\varepsilon_{A}\left(\rho_{c}\right)$ decreases from the maximum, reaches a minimum value and then increases. The minimum of $\varepsilon_{A}\left(\rho_{c}\right)$ occurs in CRR. It is interesting to note that CRs for both contrasts are the same in clear sky, i.e., 0.13 as well as in hazy sky, i.e., 0.17 . As discussed above, it is because CR increases as $\tau_{a}$ increases for low $\rho_{b}$ (Fig. 2a). The CR is around 0.09 when $\tau_{a}$ changes from 0.2 to 0.6 , and it is around 0.14 when $\tau_{a}$ changes from 0.6 to 1.0. This is because for low $\rho_{b}$, a lower value of $\mathrm{CR}$ can be enough to balance the increase in atmospheric reflectance and the decrease in surface reflection as $\tau_{a}$ increases in clear sky; however, an increase in atmospheric reflectance due to the increase of $\tau_{a}$ should be compensated with the larger attenuation of surface reflection, i.e., larger value of CR, in hazy sky. Since the behavior of $\varepsilon_{A}\left(\rho_{c}\right)$ due to $\delta \tau_{a}$ as a function of $\rho_{c}$ for different contrasts and haziness in the green band is similar to that in the red band (Fig. 5), its figure is omitted. However, quantitative analysis is reported. For a DT, $\varepsilon_{A}\left(\rho_{c}\right)$ increases as $\varepsilon_{A}\left(\tau_{a}\right)$ increases from clear sky to hazy sky for given contrast and/or from LC to MC for given haziness (Table 1). For a BT in the green band, i.e., $\rho_{c}=0.16 \sim 0.21$ (Lyapustin and Kaufman 2001), $\varepsilon_{A}\left(\rho_{c}\right)$ remains relatively stable from clear sky to hazy sky for given contrast for nadir view; however, it also increases as $\varepsilon_{A}\left(\tau_{a}\right)$ increases for $\theta_{v}$ at $45^{\circ}$. In the red band, $\varepsilon_{A}\left(\rho_{c}\right)$ remains relatively stable from clear sky to hazy sky for given contrast. For a BT, $\varepsilon_{A}\left(\rho_{c}\right)$ also increases with increased $\varepsilon_{A}\left(\tau_{a}\right)$ for given haziness in both bands. For nadir view, $\varepsilon_{A}\left(\rho_{c}\right)$ ranges from 0.010 for LC in clear sky to 0.111 for MC in hazy sky for a DT and is still greater than 0.01 for $\mathrm{BT}$ at $\mathrm{MC}$ in the green band. In the red band, it varies from 0.008 to 0.084 for a DT and is also greater than 0.01 for a BT at MC. The values of $\varepsilon_{A}\left(\rho_{c}\right)$ for a DT in both bands are larger than that for a BT with given contrast and haziness. The result of $\varepsilon_{A}\left(\rho_{c}\right)$ at $\theta_{v}=45^{\circ}$ will be discussed later. The values of $\varepsilon_{R}\left(\rho_{c}\right)$ for DTs are larger than $44 \%$ and can be up to $481 \%$ for MC in hazy sky in the green band. In the red band, they are larger than $35 \%$ and can also be up to $370 \%$ for MC in hazy sky. However, they are smaller than $8 \%$ for BTs in both bands. Quantitatively, $\varepsilon_{A}\left(\rho_{c}\right)$ can be only negligible for BTs at LC. For a DT and MC in hazy sky at nadir view, $\varepsilon_{A}\left(\rho_{c}\right)$ can be very significant, i.e., 0.111 and 0.084 , in green and red bands, respectively.

To see the effect of $\theta_{v}$ on $\varepsilon_{A}\left(\rho_{c}\right), \varepsilon_{A}\left(\rho_{c}\right)$ in the red band is plotted as a function of $\rho_{c}$ for different $\theta_{v}$ and haziness at MC (Fig. 6). The value of $\phi$ is $0^{\circ}$. Since the behavior of $\varepsilon_{A}\left(\rho_{c}\right)$ for $\mathrm{LC}$ is similar to that for MC, its figure is omitted. For $\theta_{v}=45^{\circ}$, the shape of $\varepsilon_{A}\left(\rho_{c}\right)$ vs. $\rho_{c}$ is similar to that for nadir. The value of CR increases as $\theta_{v}$ increases for given contrast and haziness. This is because as $\theta_{v}$ increases, atmospheric transmittance decreases, and surface reflectance should be increased to balance the increase in atmospheric reflectance. This physical mechanism is quite similar to the increase in CR with increasing $\tau_{a}$ for a given contrast, as discussed above (Fig. 5). The values of $\varepsilon_{A}\left(\rho_{c}\right)$ and $\varepsilon_{R}\left(\rho_{c}\right)$ for $\theta_{v}=45^{\circ}$ are also listed in Table 1. Again, $\varepsilon_{A}\left(\rho_{c}\right)$ of a DT is larger than that of a BT for $\theta_{v}=45^{\circ}$ in both bands, which is similar to the case at nadir view. For a DT, the values of $\varepsilon_{A}\left(\rho_{c}\right)$ range from 0.013 for LC in clear sky to 0.176 for $\mathrm{MC}$ in hazy sky in the green band, and they vary from 0.01 to 0.125 in the red band. For a BT, $\varepsilon_{A}\left(\rho_{c}\right)$ is negligible, i.e., less than 0.01 , for LC and still larger than 0.01 for 


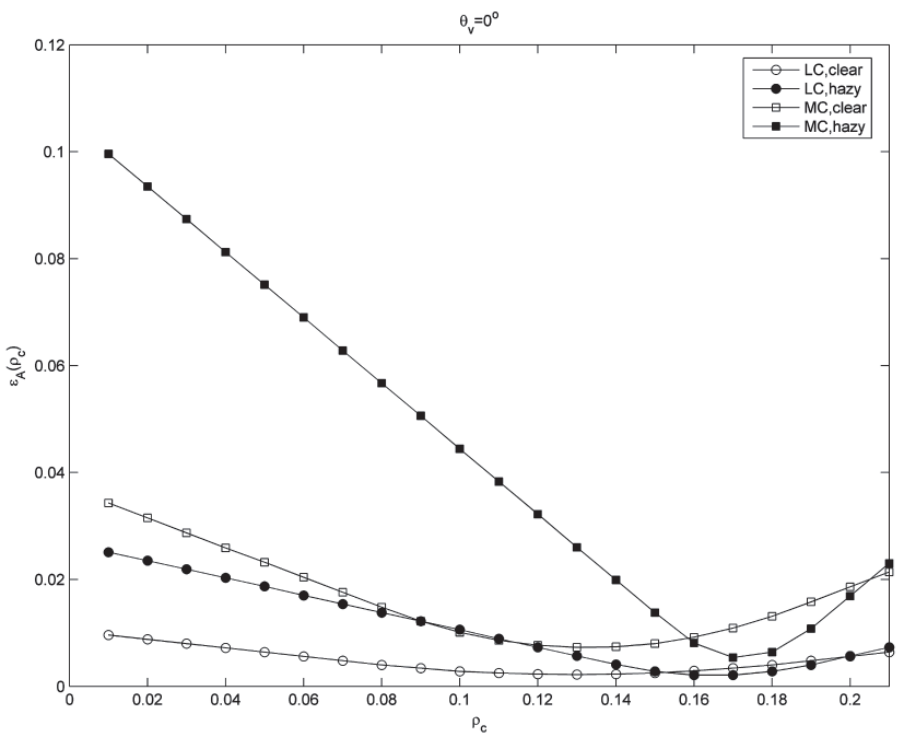

Fig. 5. Mean absolute errors of retrieved surface reflectance $\varepsilon_{A}\left(\rho_{c}\right)$ due to the errors of retrieved AOD for different contrasts and haziness as a function of $\rho_{c}$ in the red band of FORMOSAT-2 RSI. The values of solar zenith angle $\theta_{s}$ and viewing zenith angle $\theta_{v}$ are $30^{\circ}$ and $0^{\circ}$, respectively.

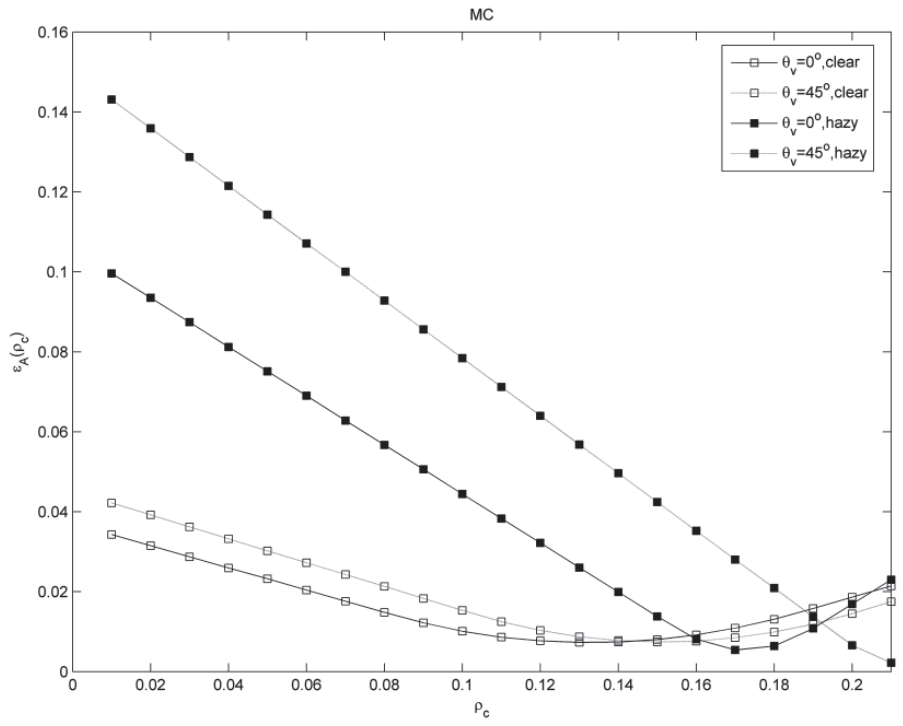

Fig. 6. Similar to Fig. 5, except for different $\theta_{v}$ and haziness at medium contrast (MC). The value of viewing azimuth angle $\phi$ is $0^{\circ}$. 
MC in both bands, especially in hazy sky in the green band. For a DT, $\varepsilon_{R}\left(\rho_{c}\right)$ is larger than $58 \%$ and can be over $700 \%$ for MC in hazy sky in the green band. Similarly, it is larger than $43 \%$ and can be over $500 \%$ for MC in hazy sky in the red band. However, it is only smaller than $30 \%$ in the green band and $10 \%$ in the red band for a BT. It is interesting to note that though $\varepsilon_{A}\left(\tau_{a}\right)$ decreases as $\theta_{v}$ increases from $0^{\circ}$ to $45^{\circ}, \varepsilon_{A}\left(\rho_{c}\right)$ increases with $\theta_{v}$ for a DT in both bands. For example, in the red band and hazy sky, $\varepsilon_{A}\left(\tau_{a}\right)$ decreases from 0.14 to 0.12 for $\mathrm{LC}$ and 0.45 to 0.38 for $\mathrm{MC}$; however, $\varepsilon_{A}\left(\rho_{c}\right)$ increases from 0.021 to 0.031 for LC and 0.084 to 0.125 for MC. Here, the decreased amount of positive bias in retrieved AOD due to AE with increasing $\theta_{v}$ should be balanced by the increased amount of surface reflectance error to compensate for both quantities: the increased atmospheric reflectance and the decreased atmospheric transmittance. In spite of similar amounts of both $\varepsilon_{A}\left(\tau_{a}\right)$ and the decrease in $\varepsilon_{A}\left(\tau_{a}\right)$ as $\theta_{v}$ increases from $0^{\circ}$ to $45^{\circ}$ in hazy sky, $\varepsilon_{A}\left(\rho_{c}\right)$ in the green band is more sensitive to $\theta_{v}$ than that in the red band. Its increase is 0.014 (from 0.027 to 0.041 ) and 0.065 (from 0.111 to 0.176 ) for LC and MC. In the red band, it is 0.010 (from 0.021 to 0.031 ) and 0.041 (from 0.084 to 0.125 ), respectively. This is because a larger increased amount of surface reflectance error is necessary to compensate the larger increased atmospheric reflectance in the green band, as $\theta_{v}$ increases. In summary, one can say that $\varepsilon_{A}\left(\rho_{c}\right)$ due to $\varepsilon_{A}\left(\tau_{a}\right)$ is more significant for a DT than that for a BT. The error in retrieved AOD due to AE can be propagated to the surface reflectance retrieval in AC. For a DT, $\varepsilon_{R}\left(\rho_{c}\right)$ is larger than $\varepsilon_{R}\left(\tau_{a}\right)$ for given contrast and haziness, especially for hazy and/or MC. Furthermore, the error propagating retrieved AOD error to retrieved surface reflectance error is increased by increasing $\theta_{v}$. For example, $\varepsilon_{R}\left(\tau_{a}\right)$ and $\varepsilon_{R}\left(\rho_{c}\right)$ are $18 \%$ and $93 \%$ for nadir, whereas they are $16 \%$ and $133 \%$ for $\theta_{v}=45^{\circ}$ for LC and hazy sky in the red band. In the green band, the increase is more significant. They are $16 \%$ and $118 \%$ for nadir and $15 \%$ and $178 \%$ for $\theta_{v}=45^{\circ}$. For a BT, $\varepsilon_{R}\left(\rho_{c}\right)$ is much smaller than $\varepsilon_{R}\left(\tau_{a}\right)$ for given contrast and haziness. This is due to the fact that the reflectance of a BT is close to CRR.

Before concluding this work, it is interesting to study the sensitivity of $\varepsilon_{A}\left(\rho_{c}\right)$ to contrast, $\tau_{a}$ and $\theta_{v}$. For a DT, as $\tau_{a}$ increases from 0.23 to 0.76 (about three times larger), $\varepsilon_{A}\left(\rho_{c}\right)$ also increases about three times for given contrast. For given haziness, as contrast increases from LC $\left(\rho_{b}=0.06\right)$ to $\mathrm{MC}\left(\rho_{b}=0.11\right)$ (only twice as large), $\varepsilon_{A}\left(\rho_{c}\right)$ increases about 3.7 times. As $\theta_{v}$ increases from $0^{\circ}$ to $45^{\circ}, \varepsilon_{A}\left(\rho_{c}\right)$ increases less than $100 \%$. Hence, one can see that $\varepsilon_{A}\left(\rho_{c}\right)$ is most sensitive to contrast, $\tau_{a}$ and least sensitive to $\theta_{v}$ for a DT, which is analogous to $\varepsilon_{A}\left(\tau_{a}\right)$. As for a BT, $\varepsilon_{A}\left(\rho_{c}\right)$ is also most sensitive to contrast in both bands. In the green band, it is sensitive to $\tau_{a}$ only for $\mathrm{MC}$ and $\theta_{v} \geq 25^{\circ}$, e.g., insignificant change at nadir (from 0.015 to 0.012 ) and significant increase of 0.036 (from 0.012 to 0.048 ) for $\theta_{v}$ at $45^{\circ}$. It is also sensitive to $\theta_{v}$ only for MC and hazy sky with a range of increase from 0.012 to 0.048 as $\theta_{v}$ increases from $0^{\circ}$ to $45^{\circ}$. However, it is insensitive to $\tau_{a}$ and $\theta_{v}$ in the red band.

\section{CONCLUSIONS}

The error in retrieved surface reflectance in AC induced by the error in retrieved AOD due to AE over a DT is studied in the green and red bands of FORMOSAT-2 RSI. The AOD is retrieved over a DT by neglecting AE. The retrieved AOD is then applied in AC to retrieve the 
surface reflectance over a wide range of surface and background reflectances. The mean absolute and relative errors of retrieved surface reflectances are determined and discussed for different contrasts, haziness and $\theta_{v}$. Simulation results show that the positive bias of the retrieved AOD due to AE over a DT can underestimate the surface reflectance of a DT and overestimate the surface reflectance of a BT in both bands. Significant errors of retrieved surface reflectance may occur except at LC for a BT. The error for a DT is larger than that for a BT for given contrast and haziness. For a DT, the error increases with $\tau_{a}$, contrast and $\theta_{v}$. It is most sensitive to contrast and least sensitive to $\theta_{v}$. For a BT, the error increases with contrast in both bands. It is sensitive to $\tau_{a}$ only for $\mathrm{MC}$ and $\theta_{v} \geq 25^{\circ}$ and to $\theta_{v}$ only for MC and hazy sky in the green band. However, it is insensitive to $\tau_{a}$ and $\theta_{v}$ in the red band. Relatively, the error of retrieved surface reflectance is enhanced by the introduced error of AOD in AC for a DT. It is suggested that $\mathrm{AE}$ be considered from AOD retrieval to surface reflectance retrieval in AC except at LC for a BT.

It is worth noting that though global observation of AOD has been successfully done by MODIS, one of the main sources of errors for the DT method is surface inhomogeneity (Chu et al. 2002; Levy et al. 2005). One might consider that this error can be testified by FORMOSAT2 RSI image in practice together with in-situ measurements and the preflight radiometric calibration of RSI. It can be identified and estimated through rigorous numerical simulation. Otherwise, it will be due to all error sources including those pertaining to pre-launch radiometric calibration coefficients and AE. Furthermore, although the in-orbit radiometric calibration has been performed very limitedly (Liu and Lin 2004), it has not also been suggested and applied by NSPO (An-Min Wu, private communication).

Acknowledgements The author is grateful to Mr. Sz-Yuan Lee at NSPO for his help of the spectral response functions of the ROCSAT-2 RSI. This work was supported by the National Science Council of Taiwan under grant NSC 94-2212-E-265-001.

\section{REFERENCES}

Butson, C. R., and R. A. Fernandes, 2004: A consistency analysis of surface reflectance and leaf area index retrieval from overlapping clear-sky Landsat ETM+ imagery. Remote Sens. Environ., 89, 369-380.

Chu, D. A., Y. J. Kaufman, C. Ichoku, L. A. Remer, D. Tanré, and B. N. Holben, 2002: Validation of MODIS aerosol optical depth retrieval over land. Geophys. Res. Lett., 29, 8007, doi:10.1029/2001GL013205.

Dubovik, O., B. Holben, T. F. Eck, A. Smirnov, Y. J. Kaufman, M. D. King, D. Tanré, and I. Slutsker, 2002: Variability of absorption and optical properties of key aerosol types observed in worldwide locations. J. Atmos. Sci., 59, 590-608.

Fraser, R. S., and Y. J. Kaufman, 1985: The relative importance of scattering and absorption in remote sensing. IEEE Trans. Geosci. Remote Sens., 23, 625-633.

Holben, B. N., D. Tanre, A. Smirnov, T. F. Eck, I. Slutsker, N. Abuhassan, W. W. Newcomb, J. Schafer, B. Chatenet, F. Lavenue, Y. J. Kaufman, J. Vande Castle, A. Setzer, B. 
Markham, D. Clark, R. Frouin, R. Halthore, A. Karnieli, N. T. O’Neill, C. Pietras, R. T. Pinker, K. Voss, and G. Zibordi, 2001: An emerging ground-based aerosol climatology: Aerosol optical depth from AERONET. J. Geophys. Res., 106, 12067-12097.

Kaufman, Y. J., and C. Sendra, 1988: Algorithm for automatic atmospheric corrections to visible and near-IR satellite imagery. Int. J. Remote Sens., 9, 1357-1381.

Kaufman, Y. J., 1989: The atmospheric effect on remote sensing and its correction. In: Asrar, G. (Ed.), Theory and Applications of Optical Remote Sensing, John Wiley \& Sons press, New York, 336-428.

Kaufman, Y. J., D. Tanre, L. A. Remer, E. F. Vermote, A. Chu, and B. N. Holben, 1997: Operational remote sensing of tropospheric aerosol over land from EOS moderate resolution imaging spectroradiometer. J. Geophys. Res., 102, 17051-17067.

Lee, Y. Y., A. M. Wu, and F. Wu, 2002: An algorithm for geometric correction of high resolution image based on physical modeling. In the $23^{\text {nd }}$ Asian Conference on Remote Sensing, 23 - 29 November 2002, Nepal.

Levy, R. C., L. A. Remer, J. V. Martins, Y. J. Kaufman, A. Plana-Fattori, J. Redemann, and B. Wenny, 2005: Evaluation of the MODIS aerosol retrievals over ocean and land during CLAMS. J. Atmos. Sci., 62, 974-992.

Liang, S., H. Fallah-Adl, S. Kalluri, J. Jaja, Y. J. Kaufman, and J. R. G. Townshend, 1997: An operational atmospheric correction algorithm for Landsat Thematic Mapper imagery over the land. J. Geophys. Res., 102, 17173-17186.

Liang, S., H. Fang, and M. Chen, 2001: Atmospheric correction of Landsat ETM+ land surface imagery-part I: Methods. IEEE Trans. Geos. Remote Sens., 39, 2490-2498.

Liang, S., H. Fang, J. T. Morisette, M. Chen, C. J. Shuey, C. L. Walthall, and C. S. T. Daughtry, 2002: Atmospheric correction of Landsat ETM+ land surface imagery-part II: Validation and applications. IEEE Trans. Geos. Remote Sens., 40, 2736-2746.

Liu, C. H., A. J. Chen, and G. R. Liu, 1996: An image-based retrieval algorithm of aerosol characteristics and surface reflectance for satellite images. Int. J. Remote Sens., 17, 3477-3500.

Liu, C. H., 2003: A simple atmospheric correction model for ROCSAT-2 RSI data. Terr. Atmos. Ocean. Sci., 14, 505-514.

Liu, C. H., 2004: A model to correct the atmospheric effect for SPOT/HRV bands. J. Photogramm. Remote Sens., 3, 15-30.

Liu, C. H., 2005: Error analysis of retrieved aerosol optical depth due to adjacency effect for ROCSAT-2 RSI bands. Terr. Atmos. Ocean. Sci., 16, 241-250.

Liu, G. R., and T. H. Lin, 2004: Pre-testing of the ROCSAT-2 RSI Absolute Radiance Calibration. In Joint Geosciences Assembly, 17 - 20 May 2004, Taiwan.

Lyapustin, A. I., 2001: Three-dimensional effects in the remote sensing of surface albedo. IEEE Trans. Geosci. Remote Sens., 39, 254-263.

Lyapustin, A. I., and Y. J. Kaufman, 2001: Role of adjacency effect in the remote sensing of aerosol. J. Geophys. Res., 106, 11909-11916.

Lyapustin, A., D. L. Williams, B. Markham, J. Irons, B. Holben, and Y. Wang, 2004: A method for unbiased high-resolution aerosol retrieval from Landsat. J. Atmos. Sci., 61, 1233-1244. 
Ouaidrari, H., and E. F. Vermote, 1999: Operational atmospheric correction of Landsat TM data. Remote Sens. Environ., 70, 4-15.

Pax-Lenney, M., C. E. Woodcock, S. A. Macomber, S. Gopal, and C. Song, 2001: Forest mapping with a generalized classifier and Landsat TM data. Remote Sens. Environ., 77, 241-250.

Pozdnyakov, D., R. Shuchman, A. Korosov, and C. Hatt, 2005: Operational algorithm for the retrieval of water quality in the Great Lakes. Remote Sens. Environ., 97, 352-370.

Sakamoto, T., M. Yokozawa, H. Toritani, M. Shibayama, N. Ishitsuka, and H. Ohno, 2005: A crop phenology detection method using time-series MODIS data. Remote Sens. Environ., 96, 366-374.

Vermote, E. F., D. Tanre, J. L. Deuze, M. Herman, and J. J. Morcrette, 1997: Second simulation of the satellite signal in the solar spectrum, 6S: An overview. IEEE Trans. Geos. Remote Sens., 35, 675-686.

Vermote, E. F., N. Z. El Saleous, and C. O. Justice, 2002: Atmospheric correction of MODIS data in the visible to middle infrared first results. Remote Sens. Environ., 83, 97-111.

Liu, C. H., 2007: Error propagation from aerosol retrieval to atmospheric correction due to adjacency effect for FORMOSAT-2 RSI band. Terr. Atmos. Ocean. Sci., 18, 827-841, doi: 10.3319/TAO.2007.18.4.827(AA). 